\title{
ANALYSIS OF AN EXPERIMENTAL SETUP FOR STRUCTURAL DAMPING IDENTIFICATION
}

\author{
Olaf P. Hentschel, Marius Bonhage, Lars Panning-von Scheidt, Jörg Wallaschek \\ Leibniz Universität Hannover, Institute of Dynamics and Vibration Research, Hannover, Germany \\ e-mail: hentschel@ids.uni-hannover.de; bonhage@ids.uni-hannover.de; panning@ids.uni-hannover.de; \\ wallaschek@ids.uni-hannover.de
}

Markus Denk, Pierre-Alain Masserey

ALSTOM Power, Steam Turbines and Generators, Baden, Switzerland; e-mail: markus.denk@power.alstom.com

In the present paper, an experimental setup for structural damping determination arising from energy dissipations within the material is presented. The experimental setup is developed in such a way that all unintended damping sources are eliminated. In this connection, priority is also given to the reproducibility of the experimental data. In addition, a vacuum chamber is developed to reduce the damping arising from the interaction with the surrounding medium. Furthermore, beam-shaped specimens are clamped in a suspended way, using screws with an apex to fix the specimens in their nodes of vibration. Then, the influence of test rig specific parameters on the damping value is analyzed. In this context, an ideal setup of the test rig is identified to measure structural damping values arising from dissipations within the material. Finally, a common model for material damping description is parameterized using the experimental data.

Keywords: damping determination, material damping, vacuum chamber, impact excitation, experimental setup

\section{Introduction}

In nearly all fields of mechanical engineering, the avoidance of High Cycle Fatigue (HCF) failures is of great importance. To reduce this risk, prediction and reduction of vibration amplitudes is a primary objective. One of the most important parameters for amplitude prediction is structural damping. In turbomachinery blading applications, for example aerodynamic, frictional and material damping are the most dominant damping mechanisms. In this paper, the latter one is in the focus of investigation.

During the last decades, the research mainly concentrated on the usage of nonlinear calculation tools to predict vibration amplitudes. Here, optimization of friction dampers in joints in dynamic systems is a superior criterion. Such calculations require specification of contact parameters as well as structural parameters. From this, many authors use low established material damping values to calculate transfer functions. Weiwei and Zili (2010) mention the material damping as low, using it for numerical blade calculation by means of a 3-D numerical contact model. Laborenz et al. (2010) also use a low material damping ratio $\xi$ with $1.41 \cdot 10^{-4}$ for establishing their eddy current approach. Using the damping ratio $\xi$ and the eigenfrequency $\omega_{0}$, the differential equation of a single degree of freedom (SDOF) system (displacement $x$ ) can be written as follows

$$
\ddot{x}+2 \xi \omega_{0} \dot{x}+\omega_{0}^{2} x=0
$$

Siewert and Stüer (2010) and Krack et al. (2012) only mention material damping as low and important for their nonlinear calculations. Petrov and Ewins (2006) use a collective loss factor $\eta$ 
for aerodynamic and material damping of 0.001. Regarding a linear SDOF-System it is possible to formulate the loss factor $\eta$ in terms of damping ration $\xi$

$$
\eta=2 \xi \sqrt{1-\xi^{2}}
$$

Resulting from the tendency to utilize blisks (blade integrated disks) to cut assembly costs and increase engine efficiency, the structural damping arising from the material is the most reasonable energy dissipation process. Therefore, quantification of mechanical damping (due to effects inside the material) is of essential relevance to predict and reduce vibration amplitudes in terms of HCF. Dealing with such low values, precise damping determination requires an appropriate experimental setup. To quantify material damping, an adequate experimental setup is elementary. For this purpose, a double reed cantilever beam is recommended as a test specimen by Gibson and Plunkett (1977), Granick and Stern (1965) as well as by Gudmundson and Wüthrich (1986). The disadvantage of using such a specimen is the necessity of frequency tuning with additional masses.

Regarding the adequate experimental setup, all other damping sources like frictional damping or damping due to the surrounding medium have to be minimized in order to identify the material damping only (see Granick and Stern, 1965; Gibert et al., 2012). Therefore, measurements under vacuum are recommended.

For the identification of damping values, Plunkett (1959) gives an overview of possible methods for single degree of freedom systems, which can be classified in time and in frequency domain approaches. Concerning multi degree of freedom systems, the Rational Fractional Polynomial method based on a parameter fit in the frequency domain is presented by Richardson and Formenti (1982). Moreover, the Least-Squares Complex Exponential method is described by $\mathrm{He}$ and $\mathrm{Fu}$ (2001). This method is classified using time domain methods. Furthermore, Bert (1973) offers an overview of methods for modeling, experimental determination and parameter identification of material damping. Within this work, the method presented by Hentschel et al. (2015) is used for damping determination.

The objective of the present work is the development of an experimental setup for material damping determination, which serves the requirements for accuracy especially for expected low damping values. The reproducibility of the experimental data is a superior criterion within the development of the experimental setup. The experimental setup including the vacuum chamber and the specimen clamping mechanism is presented, documenting the constructional effort taken to minimize sources of unintended damping, as frictional damping within joints. Then the specimen excitation using a voice coil actuator and the data acquisition process are described. An automated impact excitation is used to enable the possibility of excitation in the vacuum chamber and to satisfy the criterion of reproducibility. A method for determining damping values including a short-term Fourier transform using resampling is briefly presented (see Hentschel et al., 2015). The used test rig, in combination with the analysis method, offers the possibility to identify the decay curve of one mode shape in order to calculate the damping values. From this, decay fitting is used (see Rice et al., 2007 and Siewert et al., 2010). Then further experimental results are presented, which investigate in addition to the influence of ambient pressure also the influence of the clamping conditions on the determined specimen specific damping value of stainless steel. Using the measured data, a common model for material damping description (see Lazan, 1968 and Szwedowicz et al., 2008) is parameterized. Conclusions are given at the end.

\section{Experimental setup}

Based on a common frequency range for turbine blades, the experimental setup is developed. Using a specific specimen length, plate-type specimens are manufactured for the frequency range of interest, corresponding to the mode shape of interest. 


\subsection{Specimen clamping}

The specimens which are used for the material damping tests are beams with defined eigenfrequencies. The sample geometry (width and thickness) is selected in such a way that a wide gap between the eigenfrequencies of the test specimens is present. To reduce the influence of parasitic damping (i.e. frictional damping due to clamping), the specimens are clamped in a suspended way in their nodes of vibration (Fig. 1) corresponding to the mode shape of interest. Bolts with an apex (Fig. 1) are used to fix the specimens depending on the analyzed mode shape. This allows an evaluation of "free" modes and prevents rigid body mode shapes. In addition, the clamping mechanism design leaves a wide frequency gap between the specimen eigenfrequencies and those of the system consisting of the clamping device and the specimen (Fig. 1).

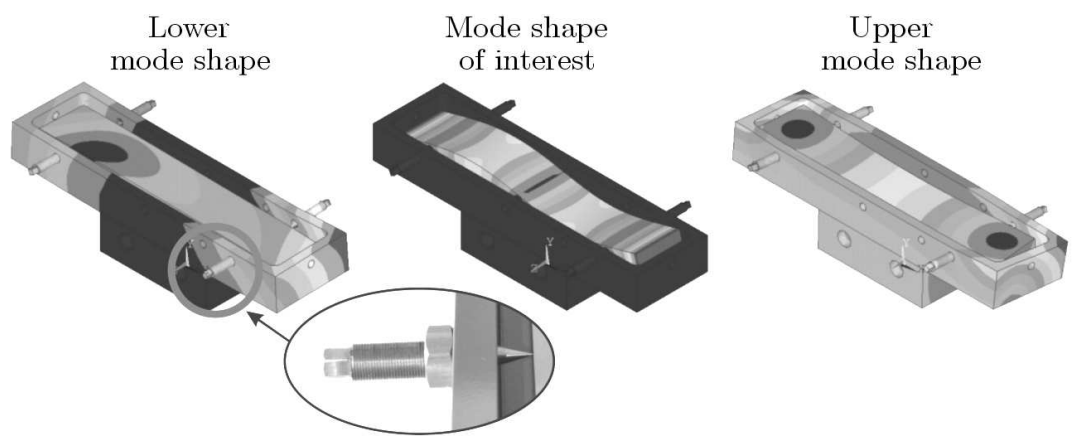

Fig. 1. Mode shapes of the clamping mechanism and the apex formed clamping screw

This avoids potential interactions between the specimen and clamping and helps one to eliminate unintended damping sources in the clamping mechanism. In Table 1, the normalized frequencies of interest $f_{s, i}$ (representing a common eigenfrequency range of turbine blades) chosen concerning the specimen and the nearest normalized eigenfrequencies of the system $f_{s y, l, i}$ and $f_{s y, u, i}$ are shown.

Table 1. Normalized frequencies

\begin{tabular}{|c|c|c|c|}
\hline Specimen & $\begin{array}{c}\text { Next lower } \\
\text { normalized } \\
\text { eigenfrequency }\end{array}$ & $\begin{array}{c}\text { Normalized } \\
\text { eigenfrequency } \\
\text { of interest }\end{array}$ & $\begin{array}{c}\text { Next upper } \\
\text { normalized } \\
\text { eigenfrequency }\end{array}$ \\
\hline \hline 1 & $f_{s y, l, a}=0.031$ & $f_{s, a}=0.106$ & $f_{s y, u, a}=0.182$ \\
\hline 2 & $f_{s y, l, b}=0.087$ & $f_{s, b}=0.32$ & $f_{s y, u, b}=0.511$ \\
\hline 3 & $f_{s y, l, c}=0.9$ & $f_{s, c}=1$ & $f_{s y, u, c}=1.08$ \\
\hline
\end{tabular}

\subsection{Excitation and measurement}

Specimens are excited by a force impact generated by a voice coil actuator. In this connection, an automated test sequence with a high reproducibility of the excitation force is realized. Based on this, a mechanical coupling of the structure and the excitation mechanism is avoided, which eliminates a potential source for data distortion. The voice coil actuator is operating in current mode. In this context, it is possible to control the acceleration of the actuator. A force sensor is used to monitor the behavior of the impact. Due to the used electrical device for specimen excitation, a high reproducibility of the impact force is realizable (see Fig. 2). Figure 2 shows in addition the relation of the preset current to the measured impact force.

The vibration is measured by a laser Doppler vibrometer. An optical measurement technique is used to avoid a coupling between structure and sensor, which may induce additional uninten- 

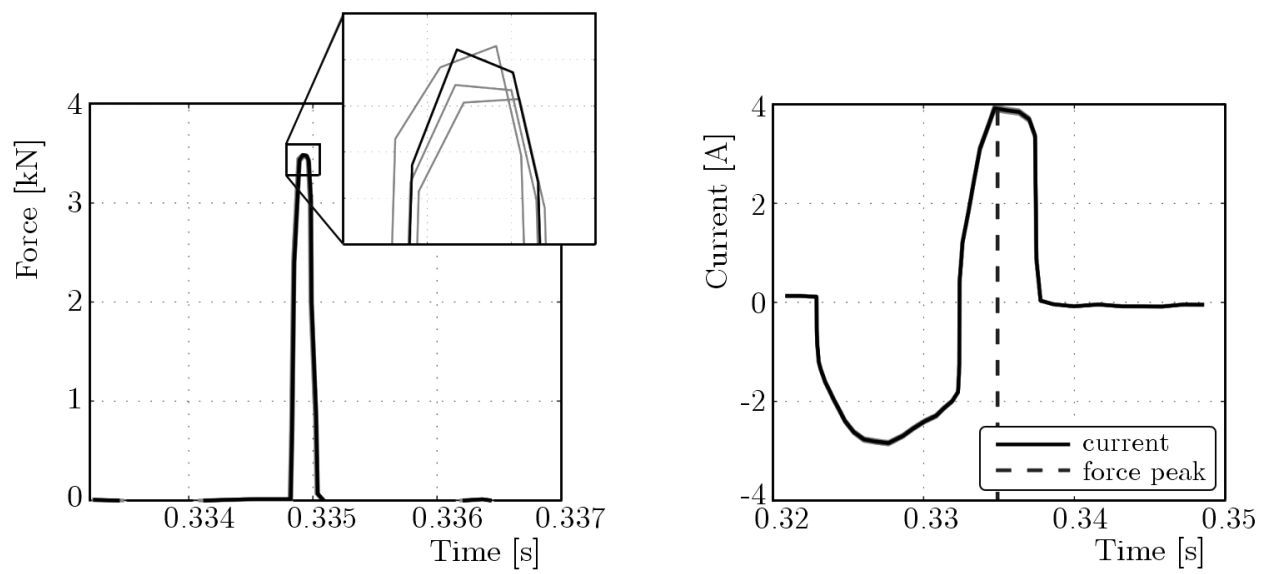

Fig. 2. Measured force with several executions of the excitation (left) and corresponding coil current (right)

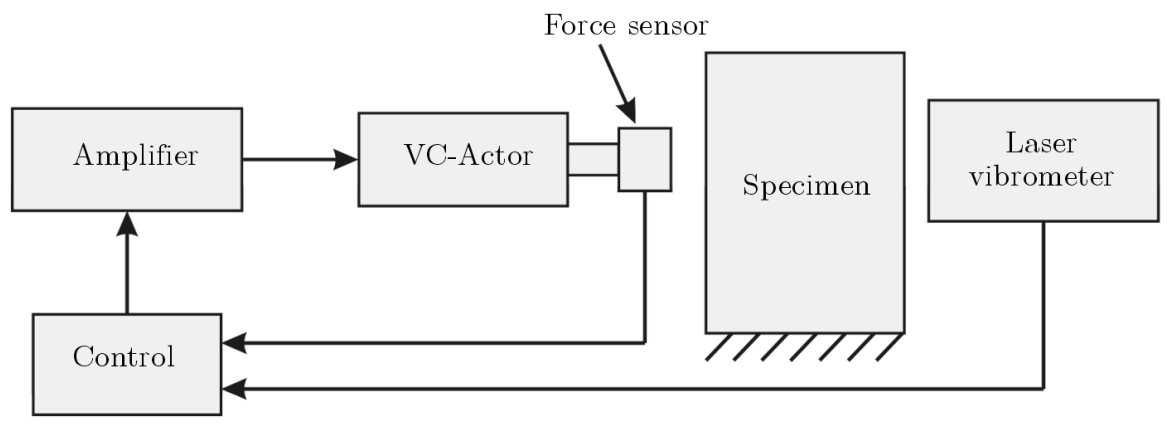

Fig. 3. Experimental setup (schematic)

ded damping. In Fig. 3, the schematic experimental setup is shown, also illustrating necessary supply and control units.

\subsection{Vacuum chamber}

The vacuum chamber is used to eliminate damping caused by the surrounding medium. The chamber is developed in two main design steps (Fig. 4). The first design step is focused on the ability to facilitate the whole experimental setup in the chamber. The specimen clamping and also the excitation mechanism are bolted to the bottom of the chamber. An electrical feed-through for the excitation mechanism and a window for vibration measurement are considered. On the basis of the first design step, the position of the flange, the flange height, the flange diameter and the wall thickness of the chamber are changed. This is ensured by frequency optimization with respect to a wide frequency range of interest (common eigenfrequency range of turbine blades). Being designed for vacuum conditions, a potential coupling of the specimen and the structure (via the remaining air in the chamber) is eliminated due to the performed frequency optimization. In this way, precise detection of the influence of the surrounding air on the damping value is also possible. Furthermore, additional masses are applicable on the chamber cap, to ensure a shift of the eigenfrequency range of the vacuum chamber. This enables a high variability of the analyzable eigenfrequencies of interest.

It is possible to define the optimization problem by maximizing the gap between the eigenfrequencies of the chamber and the eigenfrequencies of interest (representative eigenfrequency range for turbine blades). In Fig. 5, the frequency gaps are graphically presented.

The optimization variable $O p t$ has to be calculated using the three frequency gaps $\left(\Delta f_{a}\right.$, $\left.\Delta f_{b}, \Delta f_{c}\right)$. In this context, it has to be ensured that the frequency gaps reach their maximal 


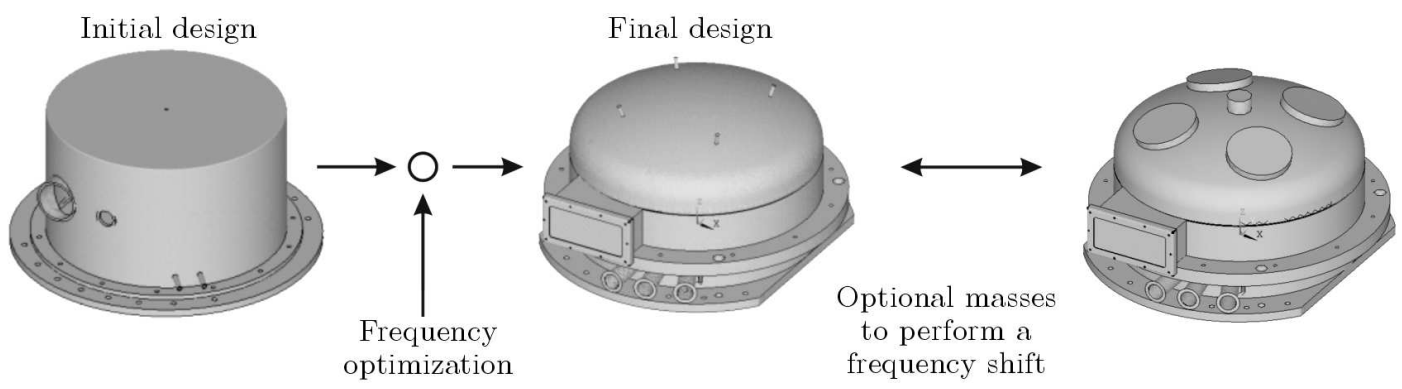

Fig. 4. Design steps of the vacuum chamber
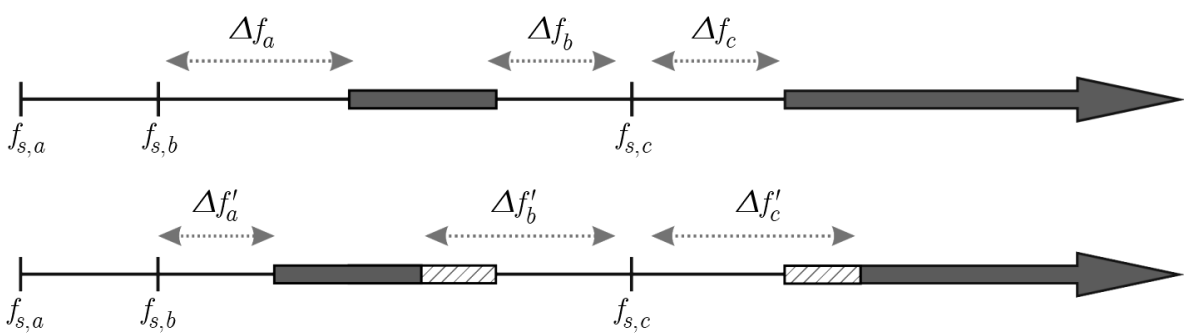

Fig. 5. Frequency gaps of the specimen and the vacuum chamber (bold: eigenfrequency range of the chamber), top: chamber without additional masses, bottom: chamber with additional masses

magnitudes. Furthermore, the magnitudes of $\Delta f_{b}$ and $\Delta f_{c}$ should be equal. As a consequence, the optimization variable can be defined as follows

$$
O p t=e \Delta f_{a}+h\left(\Delta f_{b}, \Delta f_{c}\right)
$$

The factor $e$ represents a weight factor concerning the first frequency gap. To satisfy the criterion of maximal and equal magnitudes of the factors $\Delta f_{b}$ and $\Delta f_{c}$, the function $h\left(\Delta f_{b}, \Delta f_{c}\right)$ is defined

$$
\begin{aligned}
& h\left(\Delta f_{b}, \Delta f_{c}\right)=a\left|\cos (\alpha) \Delta f_{b}-\sin (\alpha) \Delta f_{c}\right| \sigma\left(\sin (\alpha) \Delta f_{c}-\cos (\alpha) \Delta f_{b}\right) \\
& \quad+b\left|\cos (\alpha) \Delta f_{b}-\sin (\alpha) \Delta f_{c}\right| \sigma\left(\cos (\alpha) \Delta f_{b}-\sin (\alpha) \Delta f_{c}\right)-c\left(\sin (\alpha) \Delta f_{b}+\cos (\alpha) \Delta f_{c}\right)
\end{aligned}
$$

The factors $a, b, c$ and $\alpha$ are additional weight factors concerning the two frequency gaps $\Delta f_{b}$ and $\Delta f_{c}$. The several weight factors are used to ensure a high variability of the optimization process. Out of this, it is possible to repeat the optimization by changing the weighting factors to give e.g. priority to another frequency gap.

The influences of these factors on the frequency gaps are summarized in Table 2 and Fig. 6 .

Table 2. Influences of the different weight factors

\begin{tabular}{|c|l|}
\hline Factor & \multicolumn{1}{|c|}{ Influence } \\
\hline \hline$a$ & weight factor concerning the magnitude of $\Delta f_{c}$ \\
\hline$b$ & weight factor concerning the magnitude of $\Delta f_{b}$ \\
\hline$c$ & symmetric weight factor concerning $\Delta f_{b}$ and $\Delta f_{c}$ \\
\hline$\alpha$ & weight factor concerning the relationship between $\Delta f_{b}$ and $\Delta f_{c}$ \\
\hline
\end{tabular}

The optimization is carried out by performing modal analysis using the finite element program ANSYS and its sub-problem approximation method (see ANSYS, 2009). The optimization process is presented in detail in Fig. 7.

Using the defined scalar optimization variable and the optimization process presented in Fig. 7, the ideal chamber geometry with a high magnitude of the frequency gaps is calculated. The 


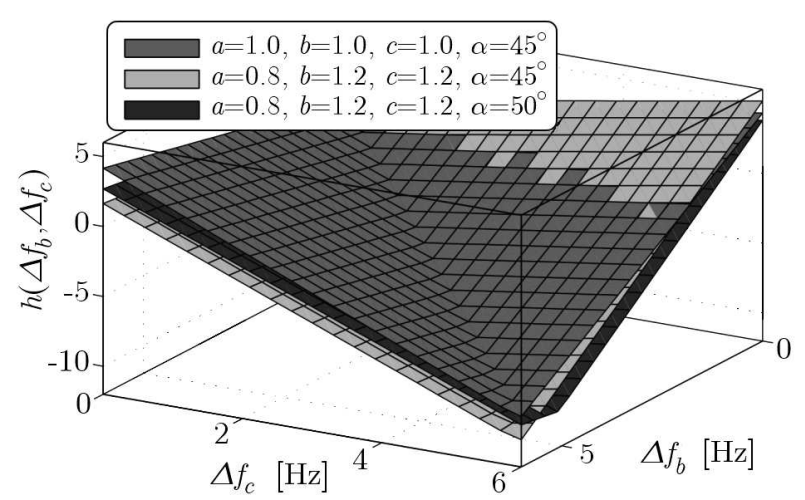

Fig. 6. Weight factors and their influences

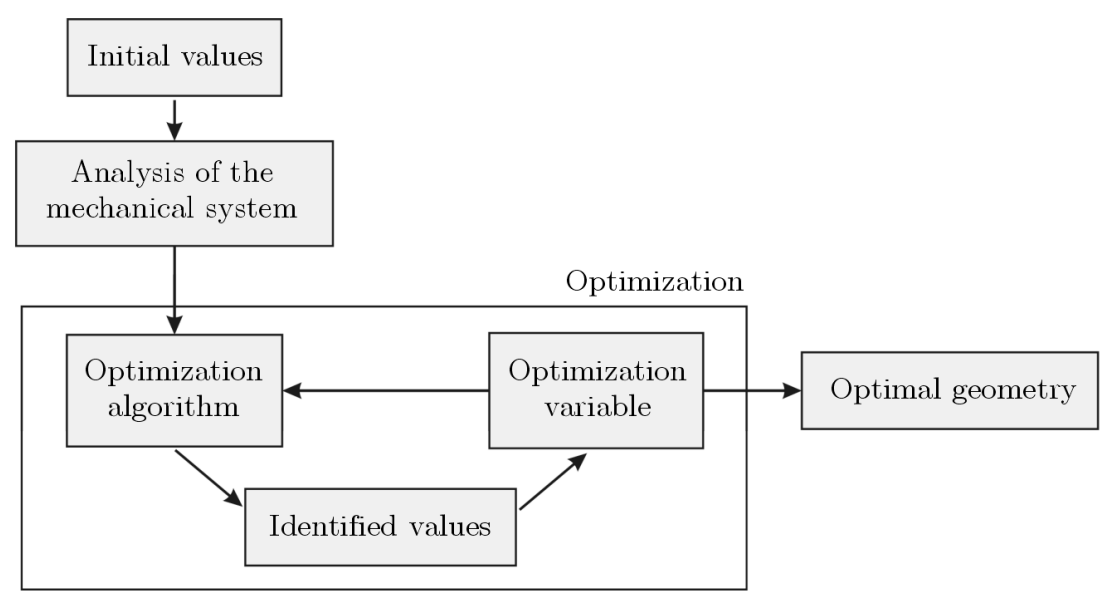

Fig. 7. Design steps of the vacuum chamber

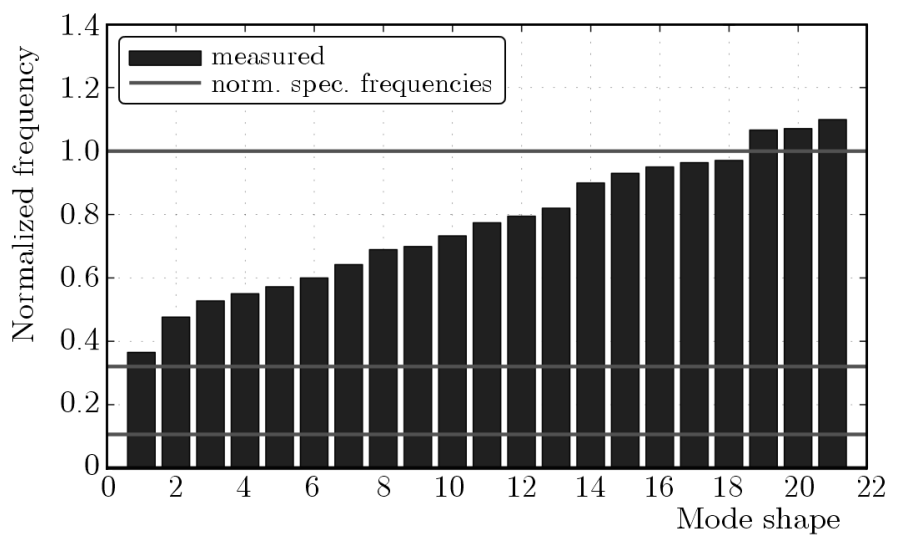

Fig. 8. Measured natural frequencies of the vacuum chamber

different eigenfrequencies measured for the manufactured chamber without additional masses are shown in Fig. 8.

It shall be pointed out that the requested frequency gaps are reached. Figure 9 shows the vacuum chamber with the integrated experimental setup.

Being equipped with a vacuum pump, low chamber pressures can be reached (see Fig. 10) and maintained during testing. 


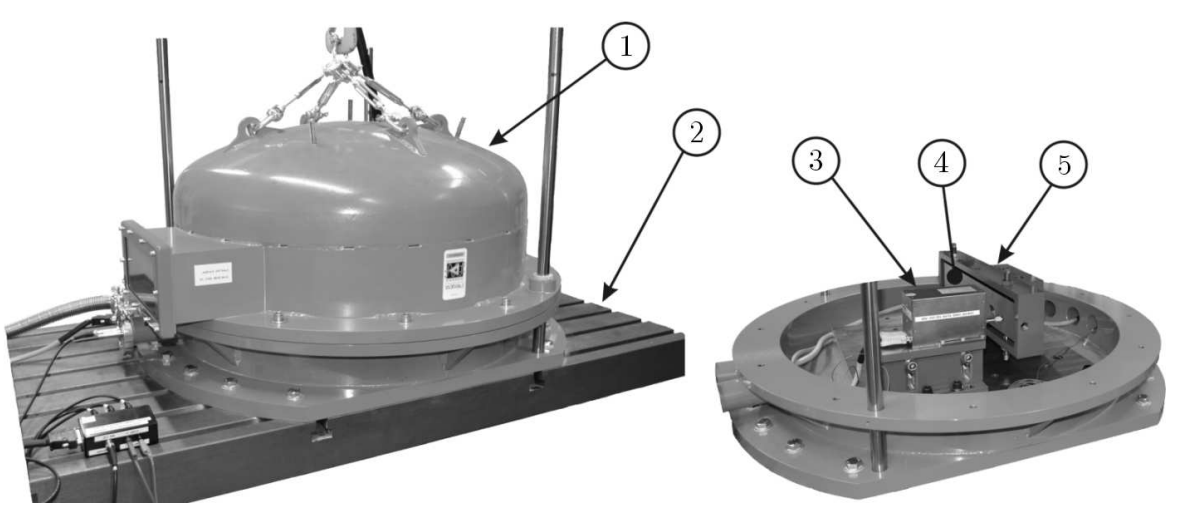

Fig. 9. Experimental setup: 1 - vacuum chamber, 2 - vibration desk, 3 - voice coil actuator, 4 - specimen, 5 - clamping device

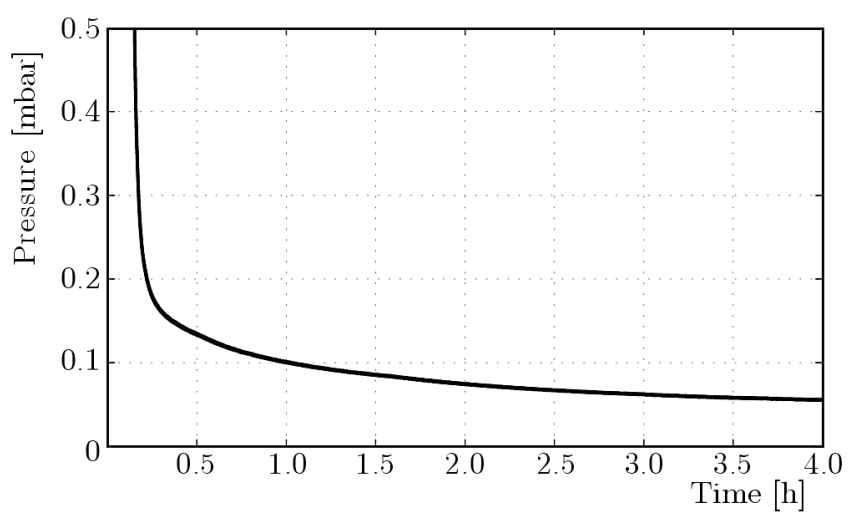

Fig. 10. Pressure-time dependency

\section{Damping determination}

The methodology of deriving damping values includes a Short-Term Fourier transform with an integrated Resampling (STFR) of the signal. The used methodology for damping identification was already presented by Hentschel et al. (2015). In this paper, only a short overview of the used methodology will be given. Using the mentioned STFR method, it is possible to get adequate information concerning vibration amplitude and eigenfrequency. This method is limited only to structures excited by a force impact (realized with the experimental setup). Here, the decaying signal $x_{u}[w]$, which is necessary for damping evaluation, is measured by a laser Doppler vibrometer. This signal can be specified by the following data sequence with $N_{w}$ values regarding the signal parts $u$

$$
x_{u}[w]= \begin{cases}x\left(t=\left(u N_{w}+w\right) T_{0}\right) & \text { for } 0 \leqslant w \leqslant N_{w}-1 \\ 0 & \text { otherwise }\end{cases}
$$

where $w$ represents the signal point and $T_{0}$ the sampling time. The signal is equidistantly divided into

$$
U=\frac{N_{S}}{N_{W}}-1
$$

parts, and it is analyzed stepwise. $N_{S}$ represents the number of all data values. In the next step, a Discrete Fourier Transform (DFT) is applied

$$
X_{u}[k]=\sum_{w=0}^{N_{w}-1} x\left(\left(u N_{w}+w\right) T_{0}\right) \mathrm{e}^{-\mathrm{j} 2 \pi \frac{k w}{N_{w}}}
$$


where $k$ describes the spectral line number. In the following, the measured signal is extracted with respect to the mode shape of interest. Using this formulation, the frequency resolution $\Delta \omega_{f}$ reads

$$
\Delta \omega_{f}=\frac{2 \pi}{T_{0} N_{w}}
$$

By analyzing weakly damped systems, the amplitude is underestimated at the eigenfrequency. To avoid this problem, resampling of the signal is performed. The goal in this context is the identification of the required sample rate in such a way that the frequency resolution $\Delta \omega_{f, n}$ is equal to an integer numbered multiple $g$ of the eigenfrequency of interest $\omega_{d \text {,int }}$.

$$
\omega_{d, \text { int }}=g \Delta \omega_{f, n}
$$

To satisfy the required sample rate corresponding to the criteria in Eq. (3.5), real numbered alternation $d$ of the sample rate $T_{0}$ is necessary

$$
\Delta \omega_{f, n}=\frac{2 \pi}{T_{0, n} N_{w}}=\frac{2 \pi}{d T_{0} N_{w}}
$$

As the eigenfrequency is unknown (due to production tolerances), optimization (such as the Nelder-Mead algorithm, Nelder and Mead, 1965) of the resampling factor $d$ is necessary. In this respect, the achievement of the maximal amplitude at eigenfrequency represents the optimization criterion.

After calculating the eigenfrequency, the modal amplitude for each part of the decay curve can be identified. Dividing the decay curve into several parts, it is possible to calculate the decay coefficient as a function of the mean amplitude.

\section{Experimental results}

In this Section, the influence of experimental setup specific parameters on the damping value is analyzed. For this purpose, an optimal setup of the test rig is identified, using the achievement of a minimal damping value as a criterion for test rig adjustment. In addition, the reproducibility of the measured data is also analyzed whereby the experimental setup is reassembled prior to each measurement. The measurements are realized four times per configuration. The tests are performed using beams with a reduced eigenfrequency for the 2-nd bending $f_{s, c}$ (see Table 1 ). As a result of this investigation, a quantification of the identified damping value concerning its quality is expected.

The first analyzed parameter is the ambient pressure. In Fig. 11, the normalized damping (ratio of the measured loss factor and the maximum measured loss factor) value versus the normalized displacement (ratio of the measured displacement and the maximum measured displacement) calculated from the measured velocity is shown.

To eliminate any possibility of an aerodynamic coupling between the vacuum chamber and the specimen, damping values are measured with and without the chamber cap under ambient conditions. Comparing the normalized damping course with and without the chamber cap (shown in Fig. 11), it can be seen, that there is no influence of the chamber cap on the measured damping value. Additionally, a decrease of the normalized damping value with decreasing pressure is visible. With regard to the ambient pressure conditions, the damping decreases to $17 \%$. The mean standard deviation is in a range of about $0.02 \%$ of the measured damping value at the maximal amplitude with respect to the analyzed configuration. Herewith, an adequate reproducibility can be obtained. Due to the used scaling factor, the influence of normalized amplitude on the damping value cannot be recognized. 


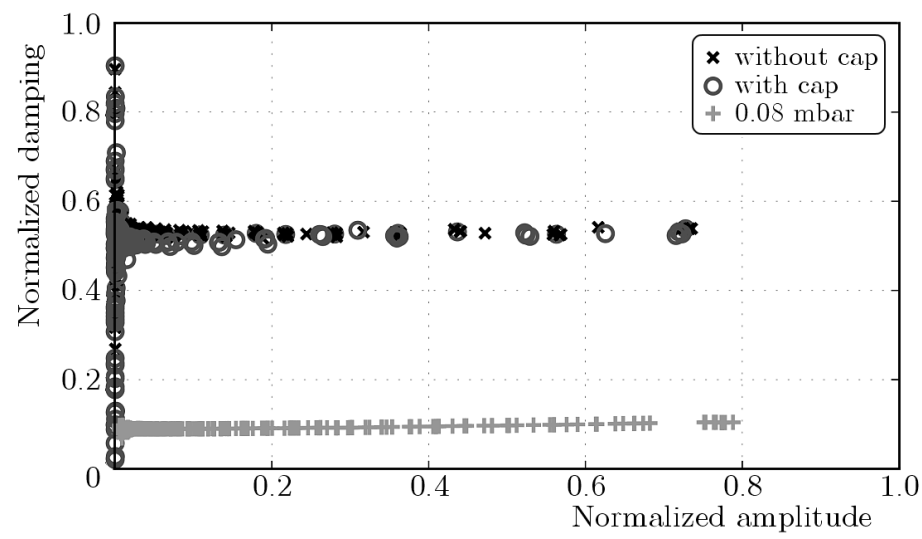

Fig. 11. Normalized damping as a function of normalized amplitude and pressure

The next analyzed parameter is the torque of the screws with the apex (see Fig. 1). This analysis is performed at 0.08 mbar (vacuum conditions). Five different torque magnitudes are analyzed. The normalized damping value versus the normalized amplitude is shown in Fig. 12.

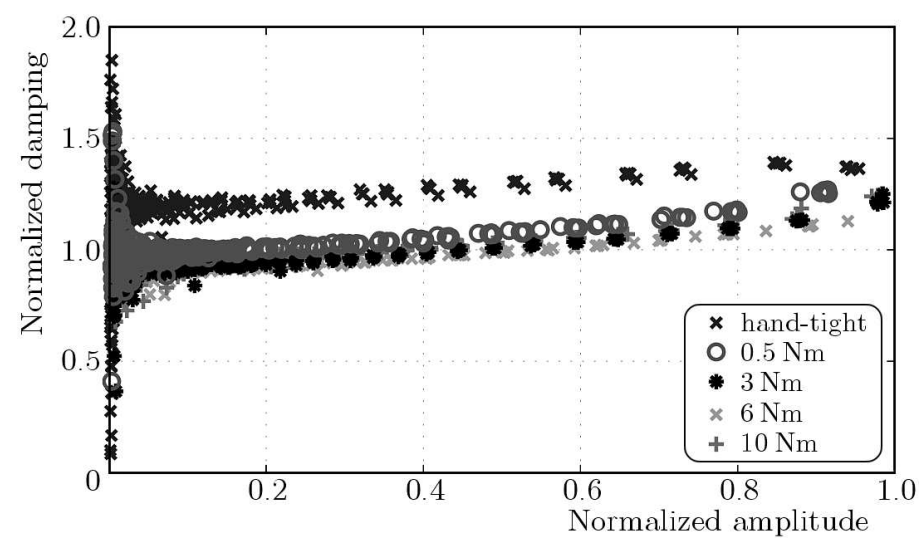

Fig. 12. Normalized damping as a function of normalized amplitude and torque

Based on this analysis, an amplitude-dependent damping value can be identified. Here, the damping value increases with the increasing amplitude. The maximal damping is measurable at the lowest torque (hand-tight). Under these conditions, the specimen is not completely fixed in its nodes of vibration, starting to rattle after the force impact. Frictional and impact effects are present and responsible for additional energy dissipations. In this respect, higher damping values are measurable. With an increasing torque magnitude, the damping value decreases to the lowest damping value at a torque magnitude of about $6 \mathrm{Nm}$. Assuming that the lowest measurable damping value course represents the energy dissipation due to dissipation within the material, the specimen-specific material damping value is measurable under these conditions. By a further increase of the torque value, the damping increases too. In this context, the apex geometry is discussed. The point of contact is plasticized, and the damaged apex lies flat on the specimen. Through these measurements, a mean standard deviation of about $0.03 \%$ of the measured damping value at the maximal amplitude with respect to the analyzed configuration is identifiable.

To check the influence of the apex variation on the damping value, different apex geometries are analyzed. From this, three different apex geometries are used (see Fig. 13), whereby the apex radius $r$ is varied.

For specimen clamping, a torque of $6 \mathrm{Nm}$ (identified before) is used. The tests are performed at 0.08 mbar (vacuum conditions). In Fig. 14, the influence of this variation on the damping value is presented. 

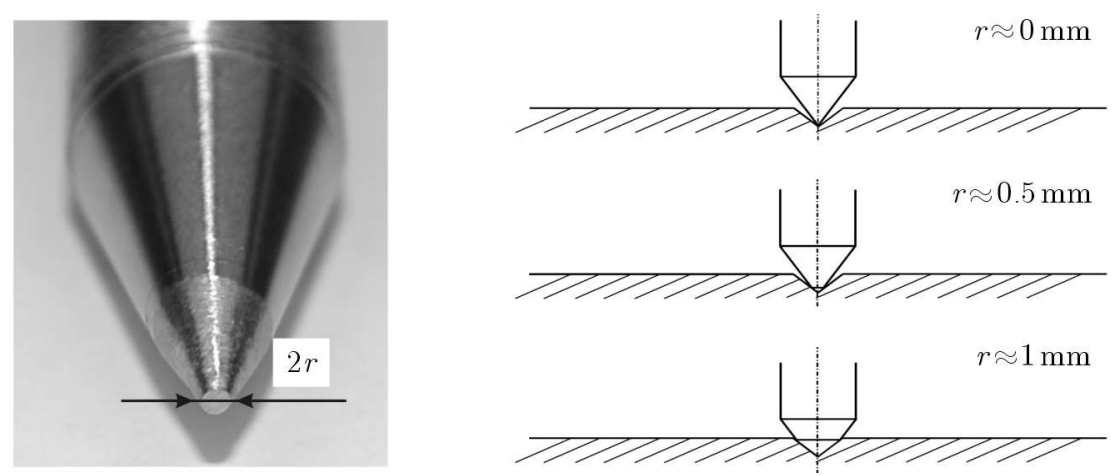

Fig. 13. Apex geometry

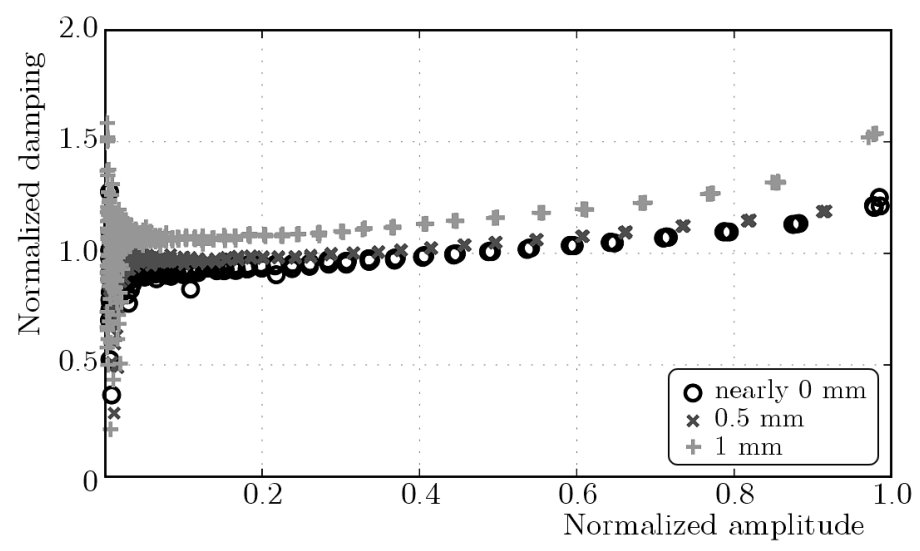

Fig. 14. Normalized damping as a function of normalized amplitude and apex geometry

The damping value increases with the increasing apex radius. This effect leads to an additional frictional damping within the contact area. Here, the lowest damping is identifiable at the lowest apex radius (approximately $0 \mathrm{~mm}$ ). For these configurations, also a low standard deviation $(0.028 \%$ of the measured damping value at the maximal amplitude) is identifiable. This is the basis for proving the adequate reproducibility of the measured values.

Based on this analysis, it can be seen that a sufficient adjustment of the experimental setup is necessary for sufficient structural damping identification arising from the material. In Table 3, the adjustment of different parameters regarding the experimental setup is summarized.

Table 3. Adjustment of the experimental setup

\begin{tabular}{|l|c|}
\hline \multicolumn{1}{|c|}{ Parameter } & Magnitude \\
\hline \hline Pressure & $0.08 \mathrm{mbar}$ \\
\hline Clamping torque & $6 \mathrm{Nm}$ \\
\hline Apex radius & $\approx 0 \mathrm{~mm}$ \\
\hline
\end{tabular}

\section{Model verification}

To demonstrate further utilization of the experimental data, the measured values are used to parameterize a common model for material damping description. For this purpose, the test rig is adjusted with respect to the data presented in Table 3. It now becomes obvious that the measured normalized damping values correspond to the lowest measured values made in previous investigations (see Fig. 14, Fig. 12 and Fig. 11). These damping values, which arise 
from dissipations within the material, are used for parameterization. In this connection, a static hysteresis model is utilized which was already recommended by Lazan (1968)

$$
\Psi_{S}=\frac{\Delta W_{S}}{2 \pi U_{S}}=\frac{1}{2 \pi U_{S}} \int_{V=0}^{V_{S}} \Delta W_{l o c} d V=\frac{1}{2 \pi U_{S}} \int_{V=0}^{V_{S}} J \sigma^{n} d V
$$

In Eq. (5.1), $\Psi_{S}, \Delta W_{S}, U_{S}$ and $V_{S}$ represent the specimen-specific loss coefficient, the entire loss, the potential energy and the entire volume of the specimen. In this context, the local energy dissipation $\Delta W_{l o c}$ is describable as a function of the local stress $\sigma$ weighted by two material constants $J$ and $n$. Analyzing the stress distribution by the Finite Element approach within the specimen, the parameters can be identified. From this, the least squares method is used to identify the magnitudes on the basis of the experimental data. In Fig. 15, the course of the normalized damping with respect to the maximal local stress $\sigma_{\max }$ is presented.

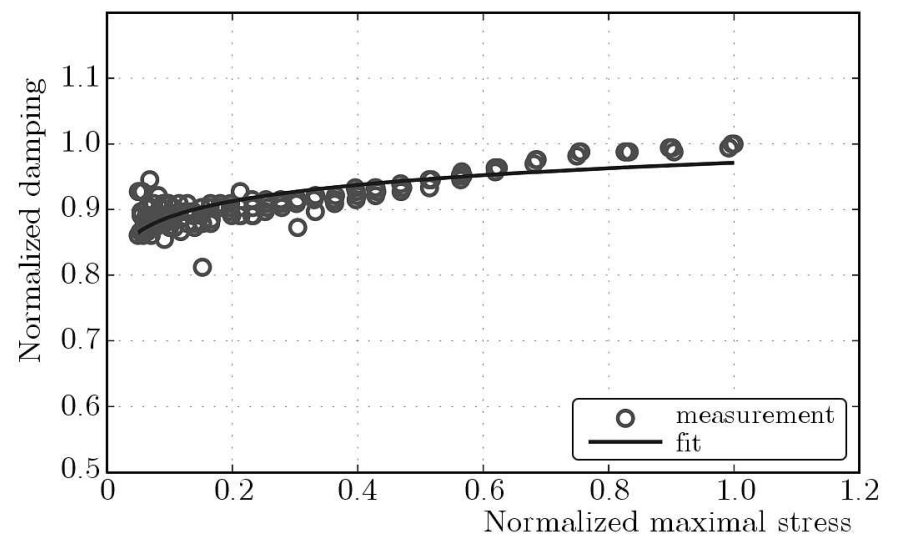

Fig. 15. Parameterization of a common model for material damping description

It is to be mentioned that the maximal local stress $\sigma_{\max }$ within the specimen corresponds to the measured amplitude. The magnitude of the maximal local stress is identified using the Finite Element approach. Finally, it can be seen that the experimental data presented in Fig. 15 are describable in a suitable way using the static hysteresis model presented by Lazan (1968). In this context, the coefficient of determination has a magnitude of $R^{2}=0.94$.

\section{Conclusions}

In the present paper, an experimental setup for specimen-specific material damping determination is developed. A design optimization with respect to the natural frequencies of a vacuum chamber and a frequency range of interest are performed. It could be shown that requested frequency gaps are achieved to avoid possible aerodynamic coupling between test specimen and vacuum chamber. In addition, test specimens are clamped in their nodes of vibration to avoid unintended frictional damping. The clamping device is also frequency optimized to avoid possible structural coupling. Moreover, a method for damping determination is discussed briefly. Subsequently, several parameters influencing the measured specimen-specific damping value using the analyzed setup are identified. It can be shown that a defined magnitude of the clamping torque as well as a defined geometry of the clamping apex is important to identify an accurate damping value. In this connection, an adjustment of the experimental setup is presented. In this context, the lowest identifiable damping value is used as a criterion for the adjustment of the experimental setup. Additionally, it can be shown that an adequate reproducibility of the 
measured values is realizable. Finally, a model for material damping description is parameterized on the basis of the measured material-specific damping values.

Using the developed experimental setup, it is possible to identify influential parameters on structural damping values arising from dissipations within the material. Based on this, development and parameterization of material damping models is realizable. It should be mentioned, that the analyzable respectively excitable frequency range is limited by the impact force.

\section{References}

1. ANSYS Inc., 2009, Advanced Analysis Techniques Guide, ANSYS Inc., Release 12.0, Canonsburg

2. Bert C.W., 1973, Material damping: an introductory review of mathematical models measures and experimental techniques, Journal of Sound and Vibration, 29, 129-153

3. Gibert C., Blanc L., Almeida P., Leblanca X., Oustyb J.P., Thouvereza F., Laîné J.-P., 2012, Modal tests and analysis of a radial impeller at rest: influence of surrounding air on damping, Proceedings of ASME Turbo Expo 2012, Power for Land, Sea and Air, Copenhagen Denmark, Paper GT2012-69577

4. Gibson R.F., Plunkett R., 1977, A forced-vibration technique for measurement of material damping, Journal of Experimental Mechanics, 17, 297-302

5. Granick N., Stern J.E., 1965, Material damping of aluminum by a resonantdwell technique, NASA Technical Note, National Aeronautics and Space Administration, Washington D.C., USA, NASA TN D-2893

6. Gudmundson P., Wüthrich C., 1986, Die Werkstoffdämpfung von Stählen bei hohen Dehnungsamplituden, Materialwissenschaft und Werkstofftechnik, 17, 286-292, Weinheim, Germany

7. He J., Fu Z.F., 2001, Modal Analysis, Butterworth Heinemann, Oxford Auckland Boston Johannesburg Melbourne New Dehli

8. Hentschel O.P., Panning-von Scheidt L., Wallaschek J., Denk M., 2015, Introduction and evaluation of a damping determination method based on a short-term fourier transform and resampling (STFR), Journal of Theoretical and Applied Mechanics, 53, 2, 395-407

9. Krack M., Panning L., Wallaschek J., Siewert C., Hartung A., 2012, Robust design of friction interfaces of bladed disks with respect to parameter uncertainties, Proceedings of ASME Turbo Expo 2012, Power for Land, Sea and Air, Copenhagen Denmark, Paper GT2010-68578

10. Laborenz J., Siewert C., Panning L., Wallaschek J., Gerber C., Masserey P.A., 2010, Eddy current damping: a concept study for steam turbine blading, Journal of Engineering for Gas Turbines and Power, 132, 1-7

11. Lazan B., 1968, Damping of Materials and Members in Structural Mechanics, Pergamon Press, Oxford London Edinburgh New York Toronto Sydney Paris Braunschweig

12. Petrov E.P., Ewins D.J., 2006, Effects of damping and varying contact area at blade-disk joints in forced response analysis of bladed disk assemblies, Journal of Turbomachinery, 128, 403-410

13. Plunkett R., 1959, Measurement of damping, [In:] Structural Damping, Ruzicka J. (Edit.), ASME, 117-131, Atlantic City NJ USA

14. Rice T., Bell D., Singh G., 2007, Identification of the stability margin between safe operation and the onset of blade flutter, Proceedings of ASME Turbo Expo 200\%, Power for Land, Sea and Air, Montreal Canada, Paper GT2007-27462

15. Richardson M.H., Formenti D.L., 1982, Parameter estimation from frequency response measurements using rational fraction polynomials, Proceedings of the First International Modal Analysis Conference, Orlando FL USA, 167-180 
16. Siewert C., Panning L., Gerber C., Masserey P.A., 2010, Numerical and experimental damping prediction of a nonlinearly coupled low pressure steam turbine blading, Proceedings of ASME Turbo Expo 2008, Power for Land, Sea and Air, Berlin Germany, Paper GT2010 51073

17. Siewert C., Stüer H., 2010, Forced response analysis of mistuned turbine bladings, Proceedings of ASME Turbo Expo 2010, Power for Land, Sea and Air, Glasgow UK, Paper GT2010-23782

18. Szwedowicz J., Secall-Wimmel T., Dnck-Kerst P., 2008, Damping performance of axial turbine stages with loosely assembled friction bolts, the non-linear dynamic assessment, Journal of Engineering for Gas Turbines and Power, 130, 3, 1-14

19. Weiwei G., Zili X., 2010, 3D numerical friction contact model and its application to nonlinear blade damping, Proceedings of ASME Turbo Expo 2010, Power for Land, Sea and Air, Glasgow UK, Paper GT2010-22292

Manuscript received January 19, 2015; accepted for print June 12, 2015 